\title{
Über das Verhalten der Milch zu Ammonsulfat und ein neues Verfahren zur Bestimmung des Milchzuckers.
}

\author{
Von \\ E. Salkowski.
}

(Aus der chemischen Abteilung des Pathologischen Instituts der Universität Berlin.)

(Der Redaktion zugegangen am 19. März 1912.)

Wenn man Milch (Kuhmilch) mit dem gleichen Volumen gesättigter Ammonsulfatlösung mischt, so wird nach Jvar Bang ${ }^{1}$ ) das Casein vollständig ausgefällt, das Filtrat enthält nur kleine, an Menge, wie es scheint, etwas wechselnde Mengen von Eiweiß.

1. $60 \mathrm{ccm}$ Milchfiltrat $=30 \mathrm{ccm}$ Milch gaben unter Zusatz von Essigsäure auskoaguliert, das Eiweiß ausgewaschen und bis zur Gewichtskonstanz getrocknet, 0,0883 g Eiweiß resp. nach Abzug von 0,0007 g Asche $0,0876 \mathrm{~g}$.

2. $70 \mathrm{ccm}$ Milchfiltrat gaben $0,1458 \mathrm{~g}$ Eiweiß, wovon 0,0009 Asche $=0,1449$ Eiweiß.

3. $75 \mathrm{ccm}$ Milchfiltrat gaben $0,1549 \mathrm{~g}$ Eiweib.

Für $100 \mathrm{ccm}$ Milchfiltrat berechnen sich danach $0,147-0,208-$ $0,206 \mathrm{~g}$ Eiweiß, für die Milch selbst also 0,294 - 0,416 - 0,414 $\mathrm{g}$ nicht durch Halbsättigung fällbares Eiweiß.

Diese Zahlen liegen ungefähr innerhalb der anderweitig für den Albumingehalt der Kuhmilch angegebenen, ${ }^{2}$ ) zum Teil höher, da nach hiesigen Bestimmungen der Albumingehalt durchschnittlich 0,3 oder etwas darüber beträgt.

Die Schnelligkeit, mit der die Mischungen aus Milch und Ammonsulfatlösung anfangs filtrieren und die absolute Klarheit der Filtrate - das Butterfett wird vollständig zurückgehalten legen den Gedanken nahe, die Fällung mit Ammonsulfat zur polarimetrischen Bestimmung des Milchzuckers zu benutzen.

1) C. Neuberg, Der Harn, sowie die übrigen Ausscheidungen usw. Berlin 1911, S. 1031.

2) Die Bestimmungen 2 und 3 sind von dem Assistenten der Abteilung Dr. E. Kretschmer ausgeführt. 
Dem steht indessen der Eiweißgehalt der Filtrate entgegen, der zwar gering ist, immerhin aber doch in Betracht kommt. Es wäre auch nicht möglich, eine Korrektur für diesen anzubringen, da die Quantität des in das Filtrat übergehenden Eiweißes etwas wechselnd zu sein scheint, eine Ausfällung des Eiweißes durch Erhitzen aber dem Verfahren den Vorzug der Einfachheit nehmen würde.

Man konnte nun vermuten, daß nach Analogie anderer Eiweißkörper auch die der Milch völlig ausgefällt werden würden, wenn man die Milch mit Ammonsulfat sättigt. Das ist nun in der Tat der Fall: Milch mit gepulvertem Ammonsulfat gesättigt, sodaß eine kleine Quantität ungelöst bleibt, liefert ein absolut klares, völlig eiweißfreies Filtrat: beim Erhitzen desselben zum Sieden mit oder ohne Zusatz von Essigsäure tritt keine Spur von Trübung ein, ebensowenig auf Zusatz von Salpetersäure mit oder ohne nachfolgendes Erhitzen.

Wollte man nun daran denken, auf dieses Verhalten ein Verfahren zur polarimetrischen Bestimmung zu begründen, so mußte zunächst der naheliegende Verdacht beseitigt werden, daß der reichliche Niederschlag Milchzucker zurückhalten könnte. $\mathrm{Ob}$ das der Fall ist, konnte man durch vergleichende Bestimmungen des Milchzuckers nach anderen Methoden feststellen, aber auch durch direkte Untersuchung des Niederschlages.

Diese führte ich folgendermaßen aus. Da die dicklichen Mischungen etwas langsam völlig abfiltrieren, verfuhr ich in allen Fällen so, daß ich die Milch zuerst mit Ammonsulfat sättigte - über die erforderliche Quantität weiter unten -, dann mit Ammonsulfatlösung soweit auffüllte, daß das Gesamtvolumen das Doppelte der angewendeten Milch betrug. Es wurden stets $50 \mathrm{ccm}$ Milch genommen und diese nach Sättigung mit Ammonsulfat auf $100 \mathrm{ccm}$ aufgefüllt. Den Niederschlag brachte ich auf ein nicht angefeuchtetes glattes Filter von ca. $16 \mathrm{~cm}$ Durchmesser, ließ völlig ablaufen und wusch den Niederschlag mit gesättigter Ammonsulfatlösung in der Weise, daß das Filter jedesmal ganz vollgegossen wurde. In der ersten Waschflüssigkeit ließ sich natürlich noch Milchzucker sowohl polarimetrisch als mit Fehlingscher Lösung nachweisen, in 
der zweiten nicht mehr. Der Sicherheit wegen wurde noch zum drittenmal gewaschen.

Es scheint mir nicht überflüssig, an dieser Stelle eine Bemerkung hinsichtlich des Nachweises des Zuckers in den ammonsulfatgesättigten Lösungen zu machen. Wenn man eine 1- oder 2\% ige Lösung von Milchzucker oder Traubenzucker mit Ammonsulfat sättigt und dann in der üblichen Weise mit Fehlingscher Lösung prüft, so bekommt man auch bei starkem Kochen und auch bei vielfacher Variation der Verhältnisse zwischen Fehlingscher Lösung und Zuckerlösung und eventuellem Wasserzusatz keine Ausscheidung von Kupferoxydul, ja auch keine Entfärbung. Eine positive Reaktion tritt erst bei Zusatz einer reichlichen Quantität starker Natronlauge ein, aber auch dann muß man einige Zeit kochen, ehe es zur Ausscheidung von Kupferoxydul kommt. Das Versagen der Reaktion mit Fehlingscher Lösung allein beruht natürlich auf der Umsetzung des Natriumhydrates mit dem Ammonsulfat bezw. der Löslichkeit des Kupferoxyduls in Ammoniak.

Der durch 3 maliges Waschen mit Ammonsulfatlösung mit Sicherheit von anhängendem Milchfiltrat befreite Filterrückstand wurde nun mit etwa 120-150 ccm Wasser behandelt. Meistens wurde dazu der Niederschlag, so gut es ging, vom Filter abgenommen und in der Reibschale mit Wasser verrieben, mitunter auch das Filter mitverrieben, die Mischung nach mehrstündigem Stehen und wiederholtem Verreiben filtriert. Das fast ganz klare, nur leicht getrübte Filtrat wurde durch vorsichtigen $\mathrm{Zu}$ satz von Essigsäure vom Casein befreit, wieder filtriert, zum Sieden erhitzt, vom Albumin abfiltriert, das Filtrat im Wasserbad eingedampft, mit Alkohol extrahiert, filtriert, eingedampft, die Alkoholbehandlung noch einmal wiederholt. Es hinterbleibt schließlich in jedem Falle eine minimale Quantität eines gelblichen .Rückstandes, der sich sehr leicht in Wasser löste. Es wurden so 5 Untersuchungen durchgeführt; nur in einem Falle war bei der Prüfung der Lösung mit Fehlingscher Lösung nach längerem Stehen am Boden des Reagenzglases ein minimaler Anflug von Kupferoxydul zu konstatieren, in 4 Fällen war die Reaktion vollkommen negativ. Die einmal gefundene minimale 


\section{Zuckerspur kommt für die quantitative Bestimmung natürlich absolut nicht in Betracht.}

Einige bezüglich des Verhaltens des Ammonsulfatniederschlages gemachte Beobachtungen kann ich nicht ganz unerwähnt lassen.

Bei der Beschreibung des Verfahrens, das ich zur Aufsuchung etwa in dem Niederschlag enthaltenen Milchzuckers eingehalten habe, habe ich gesagt, daß das Casein aus dem Filtrat durch vorsichtigen Zusatz von Essigsäure ausgefällt wurde. So war es bei dem 2., 3. und 4. der angestellten 5 Versuche, beim 1. und 5. dagegen nicht. Bei dem ersten, ganz schwach opalisierenden Filtrat war ein Niederschlag durch Essigsäure überhaupt nicht $\mathrm{zu}$ erhalten: es änderte sein Aussehen nicht.

Im 5. Versuch blieb das leicht getrübte Filtrat bei Essigsäurezusat\% anscheinend ganz unverändert, nach 48 stündigem Stehen in der Kälte hatten sich aber feinste Flöckchen in minimaler Quantität ausgeschieden, von denen abfiltriert wurde.

Beim Erhitzen zum Sieden gab das Filtrat von Nr. 1 ein anscheinend reichliches Koagulum. Die Quantität erwies sich zwar geringer, als es den Anschein hatte, betrug aber immerhin 0,203 g; da $50 \mathrm{ccm}$ Milch angewendet waren, würde dieses für $100 \mathrm{ccm} 0,406 \mathrm{~g}$ ergeben. Dabei ist aber zu bemerken, daß der Ammonsulfatniederschlag sicher bei weitem nicht quantitativ extrahiert, auch nicht nachgewaschen war. Ebenso verhielt sich das Filtrat von Nr. 5, es enthielt, wie gesagt, nur eine Spur von durch Essigsäure ausfällbarem Casein und 0,1726 durch Koagulieren fällbares Eiweiß = 0,3452 für $100 \mathrm{ccm}$ Milch.

Dagegen betrug in Nr. 2 und 3, in denen Casein reichlicher vorhanden war, das durch Erhitzen ausfällbare Eiweiß nur 0,0996 resp. $0,1005 \mathrm{~g}=0,1892$ resp. 0,201 für $100 \mathrm{ccm}$ Milch.

Da die stets aus derselben Quelle bezogene Milch ca. 0,3\% Albumin (+ Globulin) $\mathrm{zu}$ enthalten pflegt oder etwas darüber (Nr. 1 u. 2 betreffen möglicherweise dieselbe Quantität Milch, eine Notiz darüber vermisse ich leider), so liegt der Gedanke nahe, daß unter Umständen das Casein eine physikalische Zustandsveränderung erleiden könnte, bei der es durch Essigsäure unfällbar wird. Dafür würde sprechen, daß sich das auskoagulierte Albumin in Nr. 1 phosphorhaltig erwies (die anderen Niederschläge sind allerdings nicht auf P-Gehalt untersucht). Der Phosphorgehalt kann nicht auf Erdphosphate zurückgeführt werden, denn die salpetersaure Lösung der Schmelze blieb eingedampft und mit Ammoniak übersättigt, beim Stehenlassen ganz klar. Dieses Vorgehen - Alkalisieren der salpetersauren Lösung mit Ammoniak, Prüfung der aufs neue mit Salpetersäure angesäuerten Lösung mit Molybdänlösung - möchte ich überhaupt empfehlen, wenn man auf organisch gebundenen Phosphor prüfen und dabei Erdphosphate ausschließen will.

Ich kehre nun zu der Frage der Verwendbarkeit der Fällbarkeit durch Ammonsulfat zur Milchzuckerbestimmung zurück. 
Wenn man die Milch mit Ammonsulfat sättigt, tritt natürlich eine Volumszunahme ein. Da es unbequem ist, diese bei der Berechnung in Betracht zu ziehen, so wurde die Mischung stets mit gesättiger Ammonsulfatlösung auf $100 \mathrm{ccm}$ aufgefüllt. Dadurch wird natürlich auch die Filtration bedeutend beschleunigt. Gegen dieses Verfahren ist nichts einzuwenden resp. nichts anderes, als was gegen alle derartigen Bestimmungen eingewendet werden kann, nämlich, daß das Trockenvolumen des Niederschlages dabei $=0$ gesetzt wird. Anders liegt dagegen die Sache, wenn etwas Ammonsulfat ungelöst bleibt. Dieser Fehler ist natürlich durch Herstellung eines bestimmten Volumens nicht auszugleichen, er bedingt fehlerhafte, zu hohe Werte. Nun ist es aber sehr schwer, die Milch vollkommen mit Ammonsulfat zu sättigen, ohne daß ein wenig davon ungelöst zurückbleibt. Es fragte sich also, ob man nicht ohne Schaden ein wenig unterhalb der zur Sättigung erforderlichen Quantität Ammonsulfat bleiben kann.

Um dieses festzustellen, habe ich eine Reihe von Versuchen - immer mit $50 \mathrm{ccm}$ Milch und Auffüllen mit gesättigter Ammonsulfatlösung auf das Volumen von 100 - mit absteigenden Mengen Ammonsulfat, von $30 \mathrm{~g}$ angefangen, angestellt. Es ergab sich dabei, daß bei Anwendung von $17,5 \mathrm{~g}$ Ammonsulfat, die sich durch Schütteln noch leicht auflösen lassen, sich die ersten minimalsten Spuren von Eiweiß in dem übrigens völlig klaren, «blanken» Filtrat zeigen, ${ }^{1}$ ) die für die polarimetrische Bestimmung des Zuckers völlig bedeutungslos sind. Diese Quantität empfehle ich also anzuwenden.

Endlich ist noch die Frage zu beantworten, ob die beim Auflösen des Ammonsulfats eintretende Temperaturerniedrigung und die dadurch bedingte Vỏlumenabnahme nicht einen Fehler in der Bestimmung verursachen könne. Direkte Temperaturmessungen ergaben nun, daß bei Anwendung von 17,5 g Ammon-

1) Das Filtrat bleibt bei Zusatz von Salpetersäure ganz klar, auch bei nachfolgendem Erhitzen. Ebenso zunächst beim Kochen unter Essigsäurezusatz, nach dem Erkalten tritt leichte Trübung ein. Es würden auch $15 \mathrm{~g}$ genügen, da auch dabei der Eiweißgehalt ganz minimal ist, der Sicherheit halber empfehle ich aber 17,5 g. 
sulfat von Zimmertemperatur die Mischung nur um $2^{0}$ kälter ist, als die angewendete Milch. Dieser Fehler dürfte also kaum in Betracht kommen. Will man sehr genau sein, so kann man ja den Zylinder, in dem die Fällung ausgeführt wird, für einige Minuten in Wasser von $40^{\circ}$ setzen oder die zur Verdünnung benutzte Ammonsulfatlösung ein wenig erwärmen; ich halte das indessen für ganz unnötig, da die äußerste Genauigkeit beim Abmessen der Milch doch nicht $\mathrm{zu}$ erreichen ist. Erstens ist die Abmessung in einem Meßzylinder an sich ja nur bis zu einem gewissen Grade genau, zweitens gestattet die undurchsichtige Beschaffenheit der Milch nicht die Ablesung des unteren Meniscus, welche allein Genauigkeit verbürgt. Dieser Fehler ist natürlich beim Abmessen im Meßzylinder größer, als beim Abmessen mit einer Pipette. Dafür ist aber der durch das Hängenbleiben der viscösen Flüssigkeit in der Pipette beim Ausfließen entstehende Fehler größer. Auf absolute Genauigkeit können derartige Bestimmungen also überhaupt nicht Anspruch machen.

Das Verfahren gestaltet sich nun also folgendermaßen.

j0 ccm Milch werden in einem womöglich etwas breithalsigen graduierten, mit Glasstöpsel versehenen Meßzylinder von $150-200 \mathrm{ccm}$ Inhalt abgemessen, $17,5 \mathrm{~g}$ Ammonsulfat hineingeschüttet und durch energisches Schütteln in Lösung gebracht. Alsdann füllt man mit gesättigter Ammonsulfatlösung auf $100 \mathrm{ccm}$ auf, mischt durch und filtriert durch ein nicht angefeuchtetes glattes Filter von etwa $16 \mathrm{~cm}$ Durchmesser (Schleicher und Schüll, Nr. 597 oder 604 resp. ein gleichwertiges Filtrierpapier). In wenigen Minuten erhält man soviel Filtrat, wie zur polarimetrischen Bestimmung erforderlich ist. Das Filtrat ist von vornherein ganz klar, es kommt kaum vor, daß die ersten Tropfen trüb sind, ich erinnere mich wenigstens nicht, dieses jemals gesehen zu haben. Man bestimmt die Drehung und multipliziert mit 2.

Macht man die Bestimmung mit einem kleinen auf Traubenzucker graduierten Halbschattenapparat, so ist, wenn man den Milchzucker als $\mathrm{C}_{12} \mathrm{H}_{22} \mathrm{O}_{11}+\mathrm{H}_{2} \mathrm{O}$ angibt, meiner Ansicht nach eine Umrechnung nicht nötig, da die spezifische Drehung des 
krystallwasserhaltigen Milchzuckers mit der des Traubenzuckers praktisch übereinstimmt $\left(52,53^{\circ}\right.$ gegen $\left.52,6^{\circ}\right)$. Will man die Angabe für wasserfrejen Milchzucker machen, so multipliziert man mit 52,53 und dividiert durch 55,46, resp. multipliziert mit 0,94717 ( $\log \cdot 97643)$.

Benutzt man einen auf Traubenzuckerprozente graduierten Apparat, so ist die ganze Bestimmung in kürzester Zeit beendigt. Meiner Ansicht nach ist das Verfahren, was Schnelligkeit und Leichtigkeit der Ausführung betrifft, selbst dem sonst sehr empfehlenswerten auf der Michaelis-Ronaschen Eisenfällung beruhenden Verfahren von Kurt Oppenheim,1) überlegen.

Wo es sich um Anstellung von Reihenversuchen handelt, wird man sich zweckmäßig besondere Meßzylinder mit breitem Hals, eventuell auch ohne Verjüngung am oberen Ende, mit eingeschliffenem Stöpsel anfertigen lassen, behufs bequemeren und leichteren Einschüttens des Ammonsulfats.

Bei Doppelbestimmungen an derselben Milch habe ich unter Anwendung des kleinen auf Traubenzucker graduierten Polarisationsapparates keine Differenzen zwischen je $2 \mathrm{Be}-$ stimmungen nachweisen können.

Dr. Kretschmer hat eine Reihe von Doppelbestimmungen und vergleichenden Bestimmungen nach anderen Methoden mit Hilfe eines großen Polarisationsapparates ausgeführt, die sehr gute Übereinstimmung ergeben haben. Aus äußeren Gründen ist Dr. K. zurzeit verhindert, die Belege mitzuteilen, dies soll demnächst geschehen.

1) Chemiker-Zeitung, 1909, Nr. 105. 